\title{
Combining Green Tea with Aerobic Exercise Reduces Central Adiposity and Serum Lipid in Sedentary Women
}

\author{
By Soreh Hoseini* \\ Parvaneh Nazarali ${ }^{\dagger}$ \\ Parichehr Hanachit \\ Niko Khosravi \\ Maria Konstantaki ${ }^{+}$
}

\begin{abstract}
Obesity is a complex disorder which is characterised by excessive accumulation of adipose tissue and is linked to many health problems including cardiovascular disease. Green tea has anti-oxidant properties and has been shown to be beneficial for reducing obesity measures such as waist to hip ratio and fat mass. Physical activity and particularly aerobic exercise has been linked with weight reduction. The aim of this study was to investigate the effect of green tea consumption and aerobic exercise on obesity measures and in particular on lipid profiles and waist to hip ratio (WHR) in sedentary women. Forty females were recruited from a population of sedentary women who had been referred by their doctor to a fitness facility. All participants signed an informed consent and were randomly assigned to either one of four intervention groups: a) aerobic exercise, b) green tea, c) aerobic exercise and green tea, and d) control. Before and after the eight-week intervention, waist to hip ratio (WHR) and lipid levels (HDL, LDL, triglycerides and cholesterol) were measured. Results showed that WHR showed no change in control but reduced significantly in the other three groups. Cholesterol and triglyceride levels remained unchanged in all groups. HDL level increased significantly in aerobic exercise and in green tea and aerobic exercise group, but remained unchanged in green tea. LDL level reduced only in green tea and aerobic exercise group with no changes in green tea. HDL:LDL changed significantly in green tea and aerobic exercise group. These findings suggest that green tea consumption alone for eight weeks at three cups per day can reduce central adiposity. However the combination of green tea consumption and aerobic exercise appears to elicit improvements in the lipid profile in addition to reducing central adiposity.
\end{abstract}

Keywords: Aerobic exercise, Green tea, Lipid profile, Waist to hip ratio

\section{Introduction}

During the past thirty years, there has been a global increase in noncommunicable diseases namely diabetes, hypertension, metabolic syndrome and cardiovascular disease, as the world is increasingly facing an "obesity epidemic". Obesity represents a serious threat to health because it increases the risk of

* Faculty of Physical Education and Sport Science, Physiology Department, Alzahra University, Tehran Iran.

$\dagger$ Senior Lecturer, Faculty of Physical Education and Sport Science, Physiology Department, Alzahra University, Tehran Iran.

* Corresponding Author. Senior Lecturer, Faculty of Biological Science, Biotechnology Department, Alzahra University, Tehran, Iran.

- Senior lecturer, Faculty of Physical Education and Sport Science, Physiology Department, Alzahra University, Tehran, Iran.

+ Senior Lecturer, Department of Advanced Health and Exercise Sciences, Faculty of Society and Health, Buckinghamshire New University, UK. 
developing other chronic diseases, such as diabetes and cardiovascular disease (PiSunyer 1993, Ross et al. 2000). Obesity can be defined as any body mass index (BMI) of $\geq 30 \mathrm{~kg} \cdot \mathrm{m}^{2}$ (Flegal et al. 1998). Obesity can shorten the lifespan and seriously affect mortality rates not only among old (Seidell et al. 1999), but also among young obesity sufferers (Fontaine et al. 2003). Overweight which is defined as a BMI of $\geq 25-29.9 \mathrm{~kg} \cdot \mathrm{m}^{2}$ (Flegal et al. 1998) results in increased blood lipids (i.e. triglycerides, total cholesterol, low density lipoprotein or LDL and high density lipoprotein or HDL). A rise in blood lipids is associated with an increased incidence of metabolic disorders such as decreased insulin sensitivity, hyperlipidemia, blood pressure, increased risk of insulin resistance, type II diabetes and cardiovascular disease (Ramezankhany et al. 2011). The leading cause of these "modern" diseases is primarily a sedentary lifestyle that incorporates low levels of physical activity coupled with an unhealthy diet based on overconsumption of high fat or convenience food and smoking (Heller et al. 1993).

Interestingly, the incidence of non-communicable diseases, and in particular obesity, seems to be growing at a faster rate in developing countries of the Middle East. In Iran, there have been a few research studies investigating the prevalence of obesity in relation to other co-morbidities such as dyslipidemia and diabetes. Between 2004 and 2005, a nationwide cross-sectional survey that investigated the anthropometry (stature and body mass) and waist circumference of 89,404 Iranian men and women with an age range of 15-65 years (mean age 39.2 years) showed that the age-adjusted prevalence of overweight or obesity (BMI $\geq 25 \mathrm{~kg} \cdot \mathrm{m}^{2}$ ) was $42.8 \%$ in men and $57.0 \%$ in women; $11.1 \%$ of men and $25.2 \%$ of women were obese $\left(\mathrm{BMI} \geq 30 \mathrm{~kg} \cdot \mathrm{m}^{2}\right.$ ), while only $6.3 \%$ of men and $5.2 \%$ of women were underweight (Janghorbani et al. 2007). This study showed that age, low physical activity, low educational attainment, marriage and residence in urban areas were strongly associated with obesity. Abdominal obesity was considerably higher in women compared to men (54.5\% vs. $12.9 \%)$ and it appeared to increase with age.

In 2007, a national surveillance study was conducted with a sample of 5,287 Iranian citizens aged 15-64 years (Esteghamati et al. 2009). This study suggested that the prevalence of obesity and central obesity was $22.3 \%$ and $53.6 \%$, respectively, whereas the prevalence of hypertriglyceridemia and hypercholesterolemia was $36.4 \%$ and $42.9 \%$, respectively. This study also showed that prevalence rates for hypercholesterolemia were higher among females and urban residents. Another study that included 403 participants of the Zoroastrian population of Yazd with a mean age of $56.9 \pm 12.8$ years reported that the total prevalence of diabetes was $26.1 \%$ (Khalilzadeh et al. 2015). This study showed that participants who presented with diabetes not only had higher fasting blood glucose, but also had higher body mass index, triglyceride level, lower high density lipoprotein level (HDL) and systolic blood pressure compared to participants who were pre-diabetic (i.e. showing impaired fasting glucose or impaired glucose tolerance) or normoglycemic. These findings showed that diabetes is not an isolated medical condition and is often accompanied by obesity, dyslipidemia and hypertension. 
According to Janghorbani et al. (2007) the categories for underweight, normal-weight, overweight, and Class I, II, and III obesity were corresponding to a body mass index (BMI) of $<18.5,18.5$ to $24.9,25$ to 29.9, 30 to 34.9, 35 to 39.9, and $\geq 40 \mathrm{~kg} \cdot \mathrm{m}^{2}$, respectively. Abdominal obesity was defined as waist circumference (WC) $\geq 102 \mathrm{~cm}$ in men and $\geq 88 \mathrm{~cm}$ in women. The age-adjusted means for BMI were $24.6 \mathrm{~kg} \cdot \mathrm{m}^{2}$ in men and $26.5 \mathrm{~kg} \cdot \mathrm{m}^{2}$ in women. For WC the age-adjusted means were $86.6 \mathrm{~cm}$ in men and $89.6 \mathrm{~cm}$ in women. In addition to WC, the waist to hip ratio (WHR) has been used as a measure of overweight. A WHR $>0.8$ is considered as a high-risk point for predicting high blood pressure and reduced performance of physical activities in both males and females (Janghorbani et al. 2007). Many researchers consider measuring the WHR as an appropriate anthropometric indicator for evaluation of obesity and its effect on physical activity and motor performances, even though anthropometric indicators have been criticised that they are affected by gender, age, race, social and economic culture (Green et al. 2004, Nazar et al. 2010). Gelber et al. (2008) evaluated the waist to height ratio (WHtR) and proposed it as the best method for strongest association with cardiovascular disease (CVD). However, Gelber et al. pointed out that any method showing higher levels of adiposity, however measured, confers increased risk of CVD.

Tea is the second most consumed beverage in the world after water (Vinson 2000). Tea is produced from the leaves of Camellia Sinensis that contains catechins and polyphenols (Hamilton-Miller 2001). Catechins are a group of natural polyphenols found in tea that have been shown to be potent hipolipemic and anti-oxidant agents in animal studies (Vinson 2000, Leung et al. 2001, Figueroa et al. 2004). Flavonoids, the result from the secondary metabolism of plants, are widely found in tea and have been shown to have many physiologic benefits for the human body (Phung et al. 2010). Delavar et al. (2008) surveyed the incidence of metabolic syndrome in middle-aged Iranians in relation to black tea consumption. They concluded that higher consumption of black tea was associated with lower risk of developing the metabolic syndrome and they also presented with lower body mass, body fat and waist circumference. On the other hand, green tea and its catechin compounds have been shown to have several pharmacological effects such as being anti-obesity and anti-diabetic. Nagao et al. (2005) showed that daily consumption of green tea containing $690 \mathrm{mg}$ catechins over a 12-week period reduced body fat. This finding suggests that the ingestion of catechins might be useful in the prevention and improvement of lifestyle-related diseases, mainly obesity. Phung et al. (2010) studied the effect of green tea consumption with and without caffeine on 1,243 individuals. They concluded that green tea with caffeine resulted in reduced WHR, subcutaneous fat, waist and body weight, but green tea without caffeine did not have any effect on anthropometric indicators. It seems the inclusion of caffeine is beneficial in reducing anthropometric indicators.

Aerobic exercise has been shown to have a significant effect on individuals' health and especially weight control as well as reducing fat and improving lipid profile. One of the most important benefits of aerobic exercise is higher levels of energy expenditure in active individuals. It has been reported that in active 
individuals, resting energy expenditure is higher than normal individuals. To perform aerobic exercise, fat stores are mobilised for energy production utilising oxygen in the process (Hanachi et al. 2010). Research has shown that overweight and obesity have a relationship with increased serum lipids and that exercise and green tea can both reduce blood lipids in isolation (Middelberg et al. 2002). However, the combined effect of green tea consumption and aerobic exercise on obesity measures is not yet known. Therefore, the purpose of this study was to ascertain the isolated effect of green tea consumption and aerobic exercise against the combined effect of green tea consumption and aerobic exercise in a single study.

\section{Methodology}

\section{Participants}

Forty females (mean \pm SD; age: $28.17 \pm 4.15$ years, body mass: $67.12 \pm 7.72$ $\mathrm{kg}$, stature: $160.0 \pm 5.05 \mathrm{~cm}$ ) were recruited from a fitness centre in Tehran where they had been referred to exercise by their doctor. All females completed a PAR-Q and signed an informed consent before participating in the study. Participants were excluded from the study if: a) they were receiving medications i.e. insulin, antiobesity, NSAIDS (non-steroidal, anti-inflammatory drugs), blood lipid lowering drugs, antibiotics or antioxidant supplements, b) they were suffering from any cardiovascular, respiratory or metabolic condition and c) if they had used alcohol or tobacco products in the three months prior to the study. The study gained approval by the Ethics Committee, Faculty of Physical Education, University of Alzahra, Tehran, Iran.

In a preliminary session that took place at the municipal sports hall of Golestan in Tehran, participants were briefed about the study's purpose and tests to be conducted. Research assistants were employed to divide the group of forty participants into four equal sized groups of ten people by random selection. Participants were assigned to: a) control group $(\mathrm{C}, \mathrm{n}=10$, no exercise and no green tea), b) green tea group (GT, $n=10$, three cups $\left.\cdot \mathrm{d}^{-1}\right)$, c) aerobic exercise $(\mathrm{AE}, \mathrm{n}=10$, three times $\cdot \mathrm{wk}^{-1}$ ) and $\mathrm{d}$ ) green tea and aerobic exercise group (GT\&AE; three cups $\cdot \mathrm{d}^{-1}$ and three times $\left.\cdot \mathrm{wk}^{-1}, \mathrm{n}=10\right)$.

\section{Study Design}

The study was a randomised control trial with a quasi-experimental design. The design was based on observing the effect of three independent variables; a) consumption of green tea, b) aerobic exercise, and c) consumption of green tea along with aerobic exercise on dependent variables (lipid profile and measures of obesity). The study employed pre- and post-intervention measurements with an eight-week intervention period in between. 


\section{Physiological Measurements}

Body mass $(\mathrm{kg})$ and stature $(\mathrm{cm})$ of participants were determined in light clothing (shorts and t-shirt) and without shoes. Portable calibrated electronic weighing scales (Beurer Co, China) and a portable stadiometer (Seca, Germany) were used. Waist circumference (WC) was measured using constant tension tape (Tan Co, Iran) at the end of a normal expiration, with arms relaxed at the sides, at the midpoint between the lower part of the lowest rib and the highest point of the hip on the mid-axillary line. A tape measure (Poya Co, Tehran, Iran) was used above the styloid bone in order to calculate measurement around the wrist and determine the structure and ideal weight for individuals (Phung et al. 2010). In order to eliminate inter-individual error all measurements were conducted by one person.

Blood lipid profile was assessed using phlebotomy blood sampling from an antecubital one day prior to commencement of the study and immediately upon completion of the eight-week intervention period. Participants were instructed to fast for 10 to 12 hours before presenting for their blood lipid profile assessments. Venous blood $(2 \mathrm{cc})$ was collected from each participant while in the seated position using capillary tubes (TPP, Swiss). The tubes were then placed in a centrifuge (Beckman Co, USA) and centrifuged at a speed of 2500 RPM for 10 minutes in order to produce serums. The serums were kept in $-70{ }^{\circ} \mathrm{C}$ to conduct biochemical assays. Triglyceride was measured by the use of enzymatic colorimetric method (Shimadzo, Japan). Cholesterol, LDL and HDL were measured by a spectrophotometer device (Cecil BioQuest, Model CE 2501, Cecil Instruments Ltd.) using specialised kits (Randox, England).

\section{Experimental Protocol}

Each group of participants performed eight weeks of their allocated program. For the aerobic exercise programme (AE), participants performed cardiovascular exercise at the Golestan gym, Tehran, Iran. The cardiovascular exercise involved large muscle groups and utilised standard cardiovascular equipment (treadmill, stepper, cross trainer, rowing machine, cycle). The cardiovascular exercise programme was designed to elicit heart rate responses at approximately $70 \%$ of maximum heart rate $\left(\mathrm{HR}_{\max }\right) . \mathrm{HR}_{\max }$ was calculated as 220 - Age as this formula can predict the variation in actual $\mathrm{HR}_{\max }$ in $95 \%$ of individuals (Gellish et al. 2007). Each participant's $70 \%$ of $\mathrm{HR}_{\max }$ intensity was calculated. Participants were then given heart rate monitors (Suunto M1, China) to wear during their aerobic exercise sessions. They were instructed to monitor the exercise intensity and observe that they are within the target heart rate intensity during their workouts. Participants' attendance to gym sessions was set at three times per week and was recorded on an attendance sheet. The green tea consumption group (GT) were instructed to consume 3 cups (each $150 \mathrm{ml}$ ) per day. Green tea used was provided in sachets. Participants had to consume one cup of green tea in the morning, a second in the afternoon and a third in the evening. The combined AE\&GT group were instructed to consume green tea at the same quantity and at the same frequency throughout the day. This group were assigned the same exercise 
intensity and received the same training in the gym as the $\mathrm{AE}$ group. The control group (C) were informed to carry on their daily routines as previously an only show up for pre- and post-intervention testing.

Immediately prior to commencing the study, all groups completed the physiological measurements (anthropometry and blood sampling). Postintervention testing took place within 48 hours of the last gym or tea drinking session. All pre- and post-intervention measurements were conducted in the same environment and by the same investigators to ensure consistency in procedures.

\section{Statistical Analysis}

Data analysis was performed using SPSS software (version 16, SPSS, Inc, Chicago, IL). Data were analysed using paired t-tests and Analysis of Variance (ANOVA) in which post-hoc comparisons were made using the Bonferoni test. The level of significance was set at $p<0.05$.

\section{Results}

\section{Anthropometry and WHR}

There were no differences in the anthropometric characteristics of age, stature and body mass between the control, green tea, aerobic exercise and combined green tea and aerobic exercise group ( $p>0.05)$. These results are shown in Table 1. For WHR, there were no changes in the control group (pre: $0.92 \pm 0.09$ and post: $0.94 \pm 0.05 ; \mathrm{p}=0.44$ ), but there were reductions in all three intervention groups (green tea: $0.93 \pm 0.07 \mathrm{v} 0.79 \pm 0.06$, aerobic exercise: $0.90 \pm 0.07 \vee 0.77 \pm 0.05$, green tea and aerobic exercise: $0.88 \pm 0.08$ $\mathrm{v} 0.76 \pm 0.05$; all at $\mathrm{p}<0.001)$. These results are shown in Figure 1.

Table 1. Anthropometric Characteristics (mean $\pm s$ ) of Participants in the Control $(C, n=10)$, Green Tea (GT, $n=10)$, Aerobic Exercise (AE, $n=10)$ and Combined Green Tea and Exercise (GT\&AE, $n=10)$ Group

\begin{tabular}{|l|c|c|c|c|}
\hline \multicolumn{1}{|c|}{ Group } & Age (y) & Body Mass $(\mathbf{k g})$ & Stature $(\mathbf{c m})$ & BMI $\left(\mathbf{k g} \cdot \mathbf{m}^{2}\right)$ \\
\hline Control (C) & $29.5 \pm 4.08$ & $66.3 \pm 12.41$ & $156.5 \pm 4.76$ & 27.7 \\
\hline Green tea (GT) & $27.3 \pm 3.75$ & $69.1 \pm 7.99$ & $160.8 \pm 5.12$ & 26.8 \\
\hline Aerobic exercise (AE) & $26.8 \pm 3.12$ & $64.2 \pm 4.13$ & $159.1 \pm 6.09$ & 25.4 \\
\hline $\begin{array}{l}\text { Green tea \& aerobic } \\
\text { exercise (GT\&AE) }\end{array}$ & $29.1 \pm 5.65$ & $68.9 \pm 6.38$ & $163.9 \pm 4.24$ & 25.7 \\
\hline
\end{tabular}

\section{Blood Lipid Profile}

For cholesterol and triglycerides, there were no differences pre- and postintervention in any of the groups. These results are shown in Table 2. For HDL $\left(\mathrm{mg} \cdot \mathrm{dL}^{-1}\right)$, there were no changes pre- and post-intervention in either the control group $(46.9 \pm 11.04 \mathrm{v} 46.5 \pm 10.11 ; \mathrm{p}=0.56)$ or the green tea group $(50.9 \pm 11.08 \mathrm{v}$ $52.3 \pm 9.35 ; \mathrm{p}=0.18)$, but there were significant increases in the aerobic exercise group $(50.1 \pm 9.18 \vee 54.3 \pm 9.2 ; \mathrm{p}=0.031)$ and the green tea and aerobic exercise 
group $(48.3 \pm 5.34$ v $52.7 \pm 7.12 ; \mathrm{p}<0.001)$. These results are shown in Figure 2 . For LDL $\left(\mathrm{mg} \cdot \mathrm{dL}^{-1}\right)$, there were no changes in the control group $(76.9 \pm 26.93 \mathrm{v}$ $76.4 \pm 27.88, \mathrm{p}=0.49)$, the green tea group $(79.5 \pm 20.12 \vee 78.1 \pm 20.02 ; \mathrm{p}=0.15)$, or the aerobic exercise group $(81.7 \pm 26.07$ v $80.0 \pm 25.05 ; \mathrm{p}=0.19)$, but there were significant reductions in the green tea and aerobic exercise group $(87.8 \pm 21.21 \mathrm{v}$ $81.6 \pm 21.21 ; \mathrm{p}=0.001)$. These results are shown in Figure 3. For LDL to HDL ratio, there were no differences in the control group $(0.68 \pm 0.27 \mathrm{v} 0.68 \pm 0.29$; $\mathrm{p}=0.40)$, the green tea group $(0.71 \pm 0.35 \mathrm{v} 0.74 \pm 0.35 ; \mathrm{p}=0.05)$ and the aerobic exercise group $(0.66 \pm 0.19 \vee 0.66 \pm 0.19 ; \mathrm{p}=0.06)$, but there were changes in the green tea and aerobic exercise group $(0.57 \pm 0.13 \mathrm{v} 0.67 \pm 0.14 ; \mathrm{p}=0.001)$ (Figure 4).

Figure 1. Waist to Hip Ratio (WHR) in the Control (C, $n=10)$, Green Tea (GT, $n=8)$, Aerobic Exercise $(A E, n=10)$ and Green Tea and Aerobic Exercise (GT\&AE, n=9) Groups

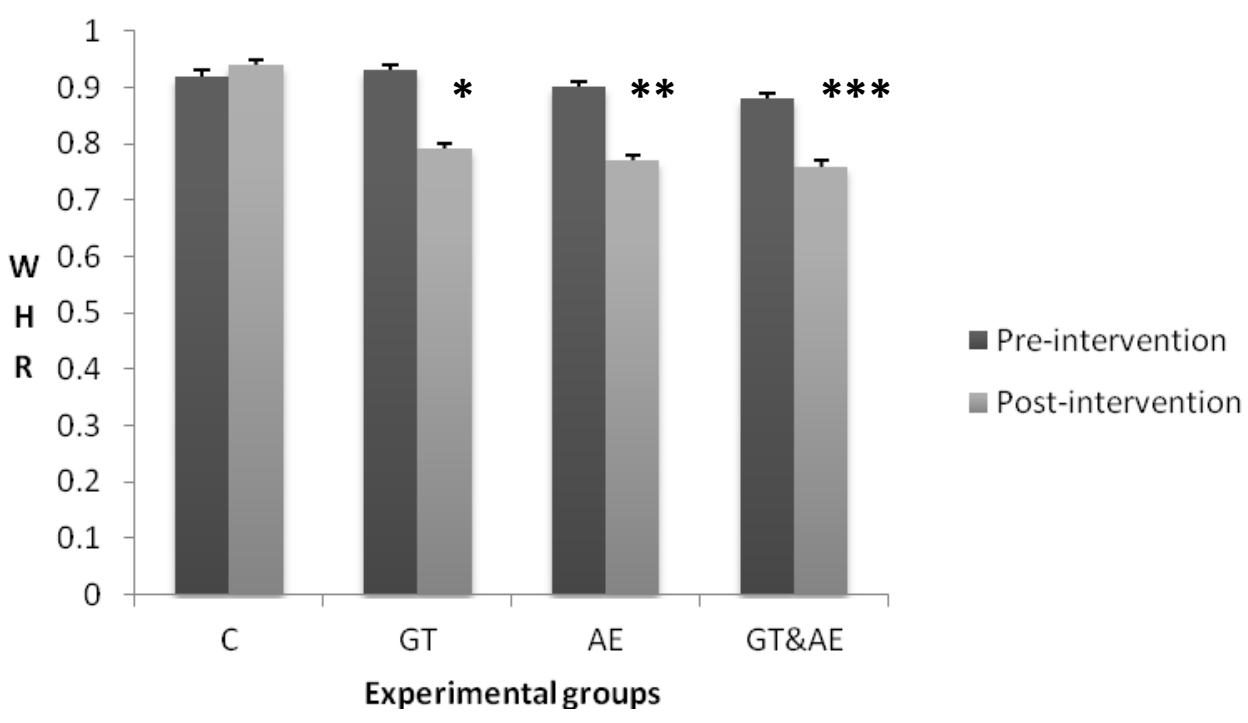

Note: Data are presented as mean $\pm s . *, * *$ and $* * *$ denote significance post-intervention in the $G T, A E$ and $G T \& A E$ group, respectively at $p<0.05$.

Table 2. Cholesterol $\left(m g \cdot d l^{-1}\right)$ and Triglyceride $\left(m g \cdot d l^{-1}\right)$ Values Pre- and PostIntervention in the Control $(C ; n=10)$, Green Tea $(G T ; n=8)$, Aerobic Exercise $(A E ; n=10)$ and Combined Green Tea and Exercise (GT\&AE, $n=9)$ Group

\begin{tabular}{|c|c|c|c|c|c|c|}
\hline & \multicolumn{3}{|c|}{ Cholesterol } & \multicolumn{3}{c|}{ Triglycerides } \\
\hline Group & Pre & Post & $\boldsymbol{p}$ & Pre & Post & $\boldsymbol{p}$ \\
\hline C & $144.1 \pm 14.19$ & $143.0 \pm 14.38$ & 0.42 & $104.4 \pm 52.42$ & $105.8 \pm 48.5$ & 0.42 \\
\hline GT & $146.4 \pm 16.51$ & $145.3 \pm 16.67$ & 0.38 & $109.1 \pm 56.51$ & $109.1 \pm 57.1$ & 1.00 \\
\hline AE & $152.9 \pm 34.32$ & $151.9 \pm 31.04$ & 0.60 & $110.0 \pm 44.29$ & $109.1 \pm 43.37$ & 0.64 \\
\hline GT\&AE & $158.3 \pm 27.95$ & $155.2 \pm 25.70$ & 0.10 & $104.8 \pm 36.92$ & $101.8 \pm 34.13$ & 0.61 \\
\hline
\end{tabular}

Note: Data were presented as mean $\pm s$. Significant differences were not detected at the $p<$ 0.05 level. 
Figure 2. High Density Lipoprotein (HDL) in the Control (C, $n=10)$, Green Tea $(G T, n=8)$, Aerobic Exercise $(A E, n=10)$ and Green Tea and Aerobic Exercise $(G T \& A E, n=9)$ Groups

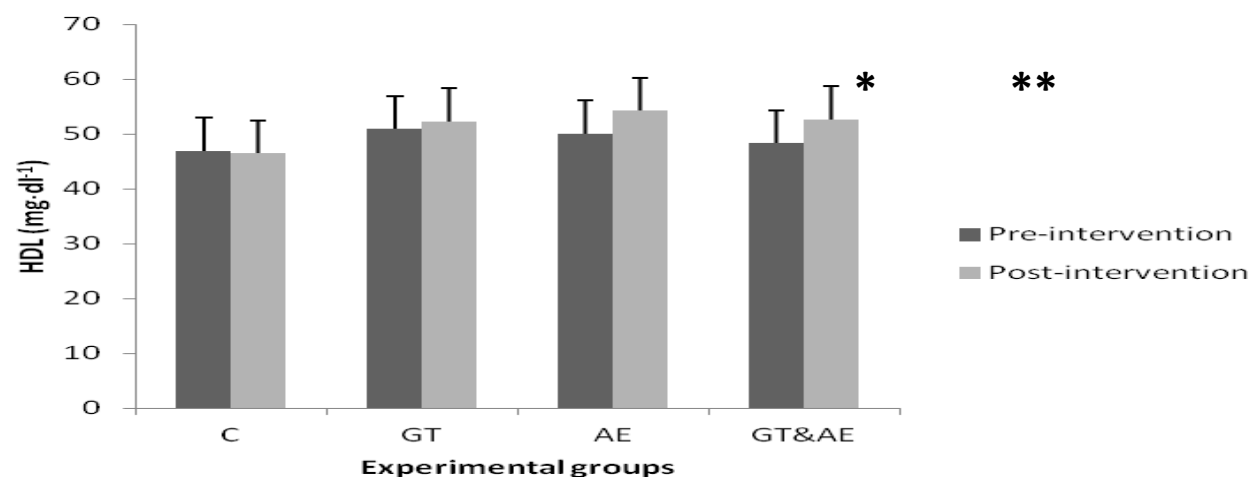

Note: Data are presented as mean and $\pm s . *$ and $* *$ denote significant difference in the $A E$ and GT\&AE group, respectively at $p<0.05$.

Figure 3. Low Density Lipoprotein ( $L D L)$ in the Control $(C, n=10)$, Green Tea $(G T, n=8)$, Aerobic Exercise $(A E, n=10)$ and Green Tea and Aerobic Exercise (GT\&AE, n=9) Groups

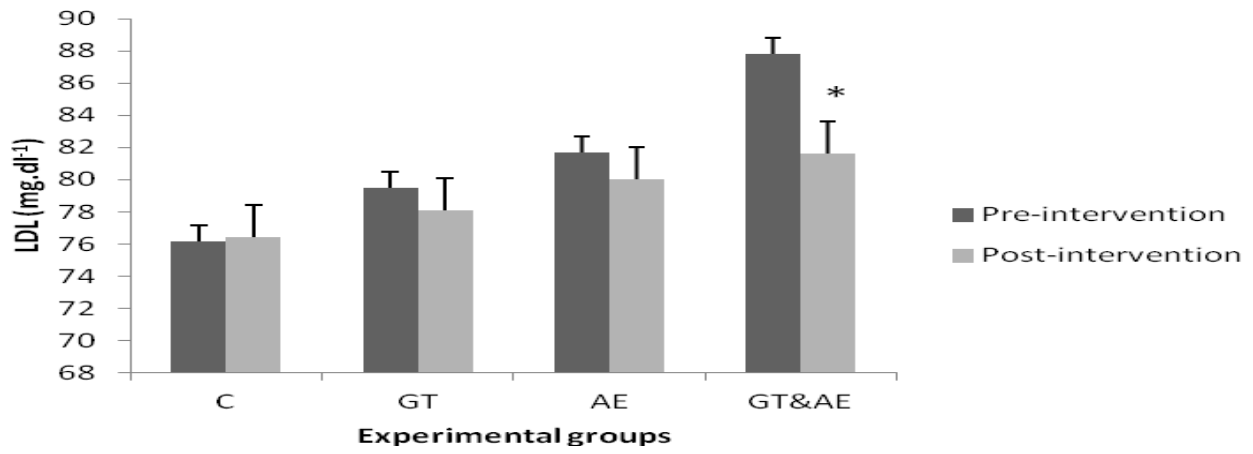

Note: Data are presented as mean and $\pm s . *$ denotes significant difference in the GT\&AE group at $p<0.05$.

Figure 4. $L D L$ to $H D L$ Ratio in the Control (C, $n=10)$, Green Tea $(G T, n=8)$, Aerobic Exercise $(A E, n=10)$ and Green Tea and Aerobic Exercise (GT\&AE, n=9) Groups

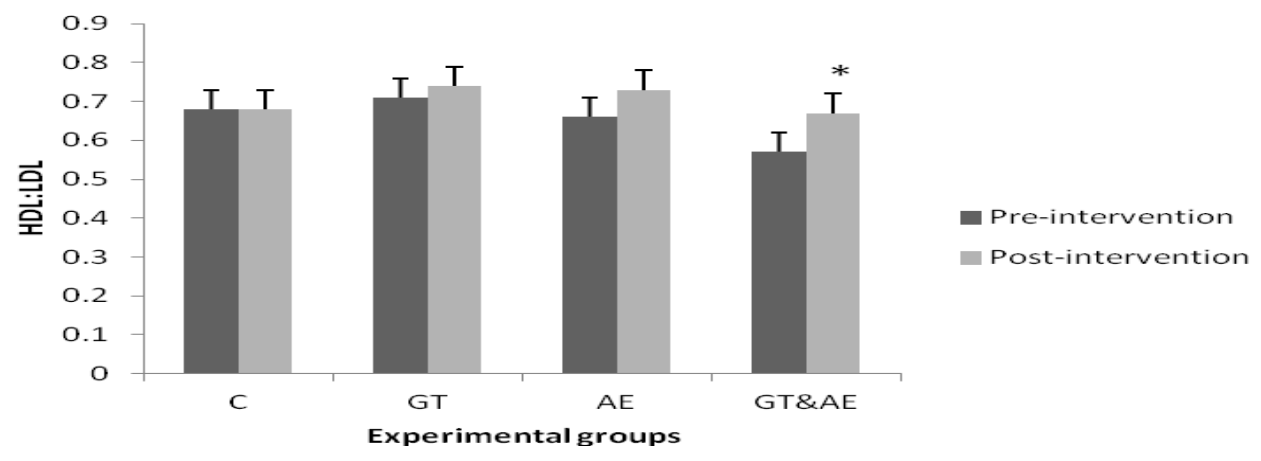

Note: Data are presented as mean and $\pm s . *$ denotes significant difference in the GT\&AE group at $p<0.05$. 


\section{Discussion}

The findings of this study demonstrated that central adiposity (as reflected by the difference in the pre- and post-intervention WHR measurements) reduced significantly in response to either green tea consumption, aerobic exercise or their combination. These results are consistent with those stated by Vasquez et al. (2007). However, no single intervention was shown to be more effective than the other two. More specifically, consumption of three cups of green tea a day for eight weeks reduced central adiposity by $15.1 \%$, aerobic exercise performed for $45 \mathrm{~min}$ a day, three days a week for eight weeks reduced adiposity by $14.4 \%$, whereas the combination of aerobic exercise and green tea ingestion reduced central adiposity by $13.6 \%$. Rexrode et al. (1998) showed that there is a link between WHR and coronary heart disease risk (CHD). In addition, a WHR of 0.88 or above is associated with an increased risk of CHD, whereas a WHR of 0.72 is associated with a reduced risk. All experimental groups in this study demonstrated an increased risk of CHD at the outset. Following the eight-week intervention, only the control group remained in the high risk category (0.94), whereas the AE, GT and AE\&GT groups showed reduced WHR (range 0.76 to 0.79 ). Interestingly, and contrary to our original hypothesis, we did not observe an augmented effect in the reduction of WHR when green tea consumption was combined with aerobic exercise.

Aerobic exercise resulted in reduced accumulation of fat in the abdominal region (central adiposity) in this study. Research evidence has shown that overweight and obesity have a positive linear relationship with increased serum lipid and that exercise can reduce blood lipids (Middelberg et al. 2002). It could be therefore stated that there is a significant correlation between WHR, change of waist circumference and serum lipid levels (Smitz and Bachl 1991). However, we did not observe a significant difference in cholesterol and triglyceride levels in the $\mathrm{AE}$ group in this study hence we cannot conclude that aerobic exercise resulted in reduced percentage of serum lipid. There was a tendency for serum lipid to reduce in the eight weeks of intervention due to aerobic exercise. Perhaps, a longer duration study would have yielded significant differences in serum lipid which would supplement the reductions in central adiposity. Previously, it has been shown that six months of running training brings about increases in the production of leptin, a hormone that is closely associated with reductions in fat mass (Kraemer et al. 2001).

Aerobic exercise results in increased density of adrenergic receptors at the cell level of fat tissue and their sensitivity toward the process of lipolysis (Vinson and Dabbagh 1998), which could explain the reductions in the indicators of abdominal obesity (waist and hip circumference). Aerobic exercise increases mitochondrial density and the activity of oxidative, electron transport chain and fat oxidation enzymes (i.e. beta-oxidation cycle enzymes and lipoprotein lipase; Vinson and Dabbagh 1998). Perhaps the lack of reduction in triglyceride in AE participants could be due to increased activity of lipoprotein lipase, increased energy expenditure and increased catabolism of lipoproteins (Saelens et al. 2007). Other factors have been implicated with reduction of blood triglyceride such as limiting 
the consumption of saturated fat, controlling carbohydrate intake, and consuming large amounts of omega-3. Also, based on the findings of Kim and Son (2009), when the initial and pre-exercise levels of triglyceride are below $120 \mathrm{mg}$, usually triglyceride does not reduce significantly. In our study, all participants had triglyceride levels below $120 \mathrm{mg}$ (range $104-110 \mathrm{mg}$ ) prior to commencement of the study. Also, in our study there were no differences identified between AE, GT and AE\&GT at levels of triglyceride which is consistent with the findings of Maki and Reeves (2009).

Green tea consumption at a frequency of three cups $(450 \mathrm{mg})$ per day resulted in significant reductions in WHR. This finding is consistent with those stated by You et al. (2006) and Nagao et al. (2005) where green tea consumed for 12 weeks reduced waist circumference, fat mass and LDL concentration. A strong positive association was also shown between reductions in body fat and LDL levels. In our study, we did not notice a change in LDL in the GT group. Our intervention lasted only six weeks however we observed a reduction in the LDL levels in the AE\&GT group. Nagao et al. (2007) performed a further study with green tea supplementation containing $583 \mathrm{mg}$ of catechins (experimental group) or green tea with just $96 \mathrm{mg}$ of catechins (control group). They found that decreases in body weight, body mass index, body fat ratio, body fat mass, waist circumference, hip circumference, visceral fat area, and subcutaneous fat area were found to be greater in the catechin group than in the control group. It appears that the amount of catechins in the green tea is an important factor that influences the magnitude of changes or reductions in measures of obesity. Even though we did not determine the amount of catechins in the green tea before administering it to our participants some reductions in obesity measures were evident.

In our study we also found higher levels of HDL in the combined green tea with aerobic exercise group and the aerobic exercise group. A previous study by Eichenberger et al. (2009) that examined the effects of a 3-week supplementation with green tea extract (GTE; $\sim 160 \mathrm{mg} \cdot$ day $^{-1}$ ) on human energy metabolism during submaximal cycling exercise also found higher resting HDL levels post-supplementation, even though they did not notice a change in LDL levels. We noticed a change in the LDL levels in our study but only in the combined green tea with aerobic exercise group. Based on previous studies, HDL is more affected by physical activity than by nutritional factors but factors such as participants' percentage of body fat, hormonal status and genetics could also affect the amount of HDL (Green et al. 2004). We observed significant difference between the groups in the amount of HDL, which is consistent with the results stated by Shashi (2012).

Other factors such as gender, age and race could have affected cholesterol levels as stated previously by Green et al. (2004). The duration of exercise could also have affected the changes in the lipid profile. In this study, the duration of each exercise session was 45 minutes and there were three sessions per week. It might have been possible to notice favourable changes in blood lipids if more than three exercise sessions per week and of longer duration than 45 minutes were used. This finding on exercise duration and its effect on 
reducing cholesterol levels is consistent with the findings stated by Kraus et al. (2002). It has also been suggested that total HDL cholesterol and LDL to HDL ratios i.e. "atherogenic indices" are risk indicators with greater predictive value of cardiovascular disease risk than isolated parameters used independently (Millán et al. 2009). In this study, it was shown that the LDL to HDL ratio was significantly different in the AE\&GT group favouring increases in HDL levels and concomitant decreases in LDL levels. This finding indicates that the lipid profile of participants in this group was positively affected by the combination of green tea consumption and regular aerobic exercise.

\section{Conclusion}

The combination of green tea consumption with aerobic exercise reduced WHR however green tea consumption alone or exercise alone had a similar effect. The inclusion of aerobic exercise did not augment this effect. Isolated serum lipid measures of cholesterol and triglyceride did not show any changes following either intervention. Nevertheless, the more sensitive indicator of cardiovascular disease risk, the LDL to HDL ratio, showed improvements only in the combined aerobic exercise and green tea consumption group. We can therefore conclude that the combination of green tea consumption and aerobic exercise is beneficial for reducing waist circumference but most importantly, it is a very effective method for reducing serum lipid and hence cardiovascular disease risk in overweight, sedentary women.

\section{Acknowledgement}

The authors would like to express their gratitude to the Faculty of Physical Education and Sport Sciences of Alzahra University, Tehran, Iran for providing financial support for this study.

\section{References}

Delavar MA, Lye MS, Syed Tajuddin BSH, Khor GL, Hanachi P (2008) Black tea consumption and risk of metabolic syndrome among middle-aged women. Research Journal of International Studies 8: 117-124.

Eichenberger P, Colombani PC, Mettler S (2009) Effects of 3-week consumption of green tea extracts on whole-body metabolism during cycling exercise in endurance-trained men. International Journal for Vitamin and Nutrition Research 79(1): 24-33.

Esteghamati A, Meysamie A, Khalizadeh O, Rashidi A, Haghazali M, Asgari F et al. (2009) Third national surveillance of risk factors of non-communicable diseases (SuRFNCD-2007) in Iran: methods and results on prevalence of diabetes, hypertension, obesity, central obesity and dyslipidemia. BMC Public Health 9(167). DOI:10.1186/1471-2458-9-167. 
Figueroa HT, Rodríguez-Rodríguez TE, Sánchez-Muniz FJ (2004) The green tea, a good choice for cardiovascular disease prevention? Archives of Latinoamerican Nutrition 54: 380-394.

Flegal KM, Carroll MD, Kuczmarski RJ, Johnson CL (1998) Overweight and obesity in the United States: prevalence and trends, 1960-1994. International Journal of Obesity and Related Metabolic Disorders: Journal of the International Association for the Study of Obesity 22(1): 39-47.

Fontaine KR, Redden DT, Wang C, Westfall AO, Allison DB (2003) Years of life lost due to obesity. Journal of the American Medical Association 289(2): 187-193.

Gelber RP, Gaziano JM, Orav EJ, Manson JE, Buring JE, Kurth T (2008) Measures of obesity and cardiovascular risk among men and women. Journal of the American College of Cardiology 52(8): 605-615.

Gellish RL, Goslin BR, Olson RE, McDonald A, Russi GD, Moudgil VK (2007) Longitudinal modelling of the relationship between age and maximal heart rate. Medicine and Science in Sports \& Exercise 39(5): 822-829.

Green JS, Stanfroth PR, Rankinen T, Leon AS, Rao DC, Skinner JS et al. (2004) The effects of exercise training on abdominal visceral fat, body composition, and indicators of the metabolic syndrome in postmenopausal women with and without estrogen replacement therapy: The HERITAGE Family Study. Clinical and Experimental Metabolism 53(9): 1192-1196.

Hamilton-Miller JMT (2001) Anti-cariogenic properties of tea (Camellia sinensis). Journal of Medical Microbiology 50(4): 299-302.

Hanachi P, Nazar AP, Rezayi N, Latifah AL (2010) Comparison of physiological and psychological wellbeing in physically active and sedentary women in Iran. Global Journal of Health Science 2(2): 249-255.

Heller DA, de Faire U, Pedersen NL, Dahlen G, McClearn GE (1993) Genetic and environmental influences on serum lipid levels in twins. New England Journal of Medicine 328: 1150-1156

Janghorbani M, Amini M, Walter C, Willett Gouya MM, Delavar A, Alikhani S et al. (2007) First nationwide survey of prevalence of overweight, underweight, and abdominal obesity in Iranian adults. Obesity 15(11): 2797-2808.

Kraemer RR, Acevedo AO, Synovitz LB, Hebert EP, Gimpel T, Castracane VD (2001) Leptin and steroid hormone responses to exercise in adolescent females runners over a 7-week season. European Journal of Applied Physiology 86: 85-91.

Khalilzadeh S, Afkhami-Ardekani M, Afrand M (2015) High prevalence of type 2 diabetes and pre-diabetes in adult Zoroastrians in Yazd, Iran: a cross-sectional study. Electron Physician 7(1): 998-1004.

Kim HJ, Son SM (2009) Effect of 12-week low calorie diet and behavior modification on the anthropometric indices and biochemical nutritional status of obese woman. Korean Journal of Community Nutritional Research Practice 3(4): 307-314.

Kraus WE, Houmard JA, Duscha BD, Knetzger KJ, Wharton MB, McCartney JS et al. (2002) Effects of the amount and intensity of exercise on plasma lipoproteins. New England Journal of Medicine 7(347/19): 1483-1492.

Leung LK, Su Y, Chen R, Zhang Z, Huang Y, Chen ZY (2001) Theaflavins in black tea and catechins in green tea are equally effective antioxidants. Journal of Nutrition 131(9): 2248-2251.

Maki KC, Reeves MS (2009) Green tea catechin consumption enhances exerciseinduced abdominal fat loss in overweight and obese adults. Journal of Nutrition 139(2): 264-270. 
Middelberg RP, Spector TD, Swaminathan R, Snieder H (2002) Genetic and environmental influences on lipids, lipoproteins and apolipoproteins: effects of menopause. Arteriosclerosis Thrombosis and Vascular Biology 22: 1142-1147.

Millán J, Pinto X, Muňoz A, Prat-Rubiès J, Pollando LF, Massana L et al. (2009) Lipoprotein ratios: physiological significance and clinical usefulness in cardiovascular prevention. Vascular Health and Risk Management 5: 757-765.

Nagao T, Komine Y, Soga S, Meguro S, Hase T, Tanaka Y et al. (2005) Ingestion of a tea rich in catechins leads to a reduction in body fat and malondialdehyde-modified LDL in men. American Journal of Clinical Nutrition 81(1): 122-129.

Nagao T, Hase T, Tokimitsu I (2007) A green tea extract high in catechins reduces body fat and cardiovascular risks in humans. Obesity 15(6): 1473-1483.

Nazar AP, Hanachi P, Rezayi N (2010) The relation of body fats, anthropometric factors and physiological functions of Iranian female national Judo team. Modern Applied Journal 4(6): 25-29.

Phung OJ, Baker WL, Matthews LJ, Lanosa M, Thorne A (2010) Effect of green tea catechins with or without caffeine on anthropometric measures. American Journal of Clinical Nutrition 91: 73-81.

Pi-Sunyer FX (1993) Medical hazards of obesity. Annals of Internal Medicine 119(7): 655-660.

Ramezankhany A, Nazar AP, Hanachi P (2011) Serum leptin concentration and some cardiovascular risk factors in response to aerobic exercise and weight loss diet in sedentary women. Hormozgan Medical Journal 159(3): 184-190.

Rexrode KM, Carey VJ, Hennekens CH, Walters EE, Colditz GA, Stampfer MJ et al. (1998) Abdominal adiposity and coronary heart disease in women. JAMA 280(21): 1843-1848.

Ross R, Dagnone D, Jones PJ, Smith H, Paddags A, Hudson R et al. (2000) Reduction in obesity and related co-morbid conditions after diet-induced weight loss or exercise-induced weight loss in men: a randomized, controlled trial. Annals of Internal Medicine 18: 92-103.

Saelens BE, Seeley RJ, Van Schaick K, Donnelly LF, O’Brien KJ (2007) Visceral abdominal fat is correlated with whole-body fat and physical activity among 8-yold children at risk of obesity. American Journal of Clinical Nutrition 85(1): 46-53.

Shashi KA (2012) Cardiovascular benefits of exercise. International Journal of General Medicine 5: 541-545.

Smitz G, Bachl N (1991) Physical training programs and their effects on aerobic capacity risk profile in sedentary individuals - design of a long term exercise training program. Journal of Sport Medicine and Physical Fitness 31: 283-293.

Seidell JC, Visscher TLS, Hoogeveen RT (1999) Overweight and obesity in the mortality rate data: current evidence and research issues. Medicine and Science in Sports and Exercise 31(Suppl.): S597-S601.

You T, Murphy KM, Lyles MF, Demons JL, Lenchik L, Nicklas BJ (2006). Addition of exercise training to dietary weight loss preferentially reduces abdominal adipocyte size. International Journal of Obesity 30(8): 1211-1216.

Vazquez G, Duval S, Jacobs DR Jr., Silventoinen K (2007) Comparison of body mass index, waist circumference, and waist to hip ratio in predicting incident diabetes: a meta-analysis. Epidemiology Reviews 29: 115-128.

Vinson JA, Dabbagh YA (1998) Effect of green and black tea supplementation on lipids, lipid oxidation and fibrinogen in the hamster: mechanisms for the epidemiological benefits of tea drinking. FEBS Letters 433(1-2): 44-46.

Vinson JA (2000) Black and green tea and heart disease: a review. Biofactors 13(1-4): 127-132. 
